\title{
Low Grade Ghondrosareoma of Bone with Unusual Metastatic Presentation
}

Karuna Singh, Omar Sadat Shah, Anshuman Dalbera', V. Sirisha Devi, Dhananjay Ghosh Departments of Radiation Oncology and Medicine', Batra Hospital and Medical Research Centre, New Delhi 1100062 , India.

\section{Abstract:}

Low grade chondrosarcoma is considered to have less aggressive clinical course and is generally managed by excision. We hereby report a diagnosed patient with low grade chondrosarcoma of right proximal femur who presented for palliative radiotherapy. PET-CT scan of whole body showed vertebral metastasis. She later developed occult blood in stool for which she was evaluated with endoscopy which revealed pedunculated ulcerative lesion in stomach. She also had colonic mass lesion and paratracheal lymph nodal enlargement. This report is to make ourselves aware the possible metastatic potential of low grade chondrosarcoma.

Key words: Bone Neoplasms, Chondrosarcoma, Colonic Neoplasms, Occult Blood, Positron Emission Tomography.

\section{Introduction}

Primary osseous low grade chondrosarcomas are considered to have low metastatic potential and least aggressive clinical course among all bone tumors. They account for $20 \%$ of all bone tumors [1]. Metastasis if occur, generally involve lungs, bones and liver. The involvement of stomach and lymph nodes in its clinical course is extremely rare. There is also paucity of literature on these uncommon lesions. Whatever literature we have is through case reports and small case series. We hereby report a case of low grade chondrosarcoma who was referred to us for palliative radiotherapy for painful bone metastasis but later progressed rapidly to develop lymph nodal enlargement, stomach metastasis, colonic mass lesion over a period of one month.

\section{Case Report}

76 years old female presented with a mass lesion in right proximal femur. MRI hip showed a well-defined lesion $5.9 \times 3.7 \times 2 \mathrm{~cm}$ in the proximal metaphyseal region of right femur extending to greater trochanter. She was evaluated and underwent resection of the right proximal femur and placement of endoprosthesis under frozen control. Post-operative histopathology reported as low grade chondrosarcoma and patient was kept on follow up. Patient remain symptom free for 6 months post-surgery when she developed pain in the lower back radiating to the right lower limb.

PET-CT evaluation was done which revealed FDG avid $2.9 \times 2.8 \mathrm{~cm}$ in size heterogenously

Corresponding Author: Dr. Karuna Singh

Email: karuna.mamc@gmail.com

Received: January 28, 2015 | Accepted: September 11, 2015 | Published Online: September 30, 2015 This is an Open Access article distributed under the terms of the Creative Commons Attribution License (creativecommons.org/licenses/by/3.0)

Conflict of interest: None declared | Source of funding: Nil | DOl: http://dx.doi.org/10.17659/01.2015.0107 
enhancing enlarged lymph node in posterior suboccipital region, $3.8 \times 3.2 \mathrm{~cm}$ heterogenously enhancing enlarged lymph node in right paratracheal region, enlarged heterogenously enhancing lymph nodes are seen in the left paraaortic, retrocaval, bilateral common iliac, right external iliac, right internal iliac and right inguinal region largest measuring $2.3 \times 1.4 \mathrm{~cm}$. A large soft tissue component measuring $8.6 \times 7 \times 10.7$ $\mathrm{cm}$ adjacent to the prosthesis extending along the right iliac blade and focal lytic sclerotic areas of involving sternum, right humerus, left clavicle, bilateral scapula, few bilateral ribs, cervicodorsal, lumbar and sacral vertebrae, bilateral pelvic bones and bilateral femora was seen [Fig.1].

Hence patient was referred to our department for palliative radiotherapy. We planned her for $30 \mathrm{GY} / 10$ fractions over a period of 2 weeks to painful sites and local recurrent lesion. During the period of radiotherapy she complained of severe pain over the post occipital region hence palliative radiotherapy was also planned to a dose of $18 \mathrm{GY} / 6$ fractions over a period of 1 week to the post-occipital lymph node. She tolerated the treatment well and was further planned for palliative chemotherapy with tab sorafenib after a gap of 1 week. Within a period of one week, patient presented in the casualty with the complaints of generalized weakness, palpitations, ghabrahat and respiratory discomfort. Laboratory parametres revealed low hemoglobin ( $\mathrm{Hb}$ 6.3), hyponatremia and deranged kidney functions. She was started on supportive management. Stool for occult blood was $4+$. She was advised for upper endoscopy evaluation which revealed a polypoidal lesion with ulcerated surface and actively oozing blood in the body of stomach [Fig.2]. Biopsy could not be taken in apprehension of risk of bleeding, sclerotherapy was done with adrenaline and biopsy deferred for later date. Contrast enhanced CT scan was done to rule out the possibility of second primary which showed soft tissue density lesion in the anterior segment of

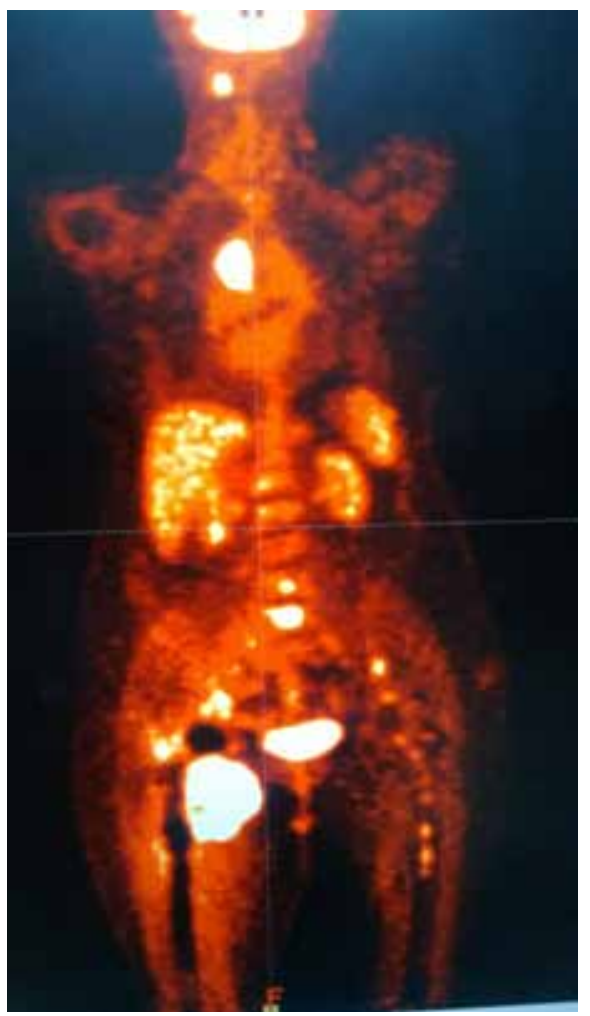

Fig.1: Soft tissue component adjacent to the prosthesis extending along the right iliac blade and focal lytic sclerotic areas of involving sternum, right humerus, left clavicle, bilateral scapula, few bilateral ribs, cervicodorsal, lumbar and sacral vertebrae, bilateral pelvic bones and bilateral femora.

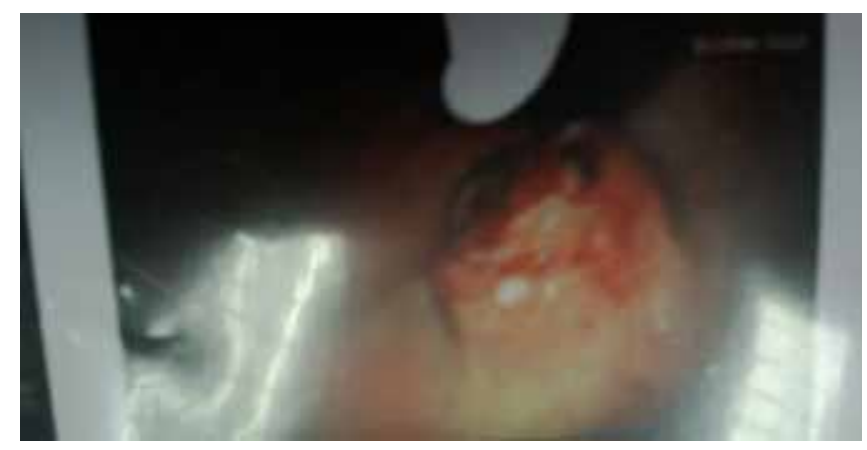

Fig.2: Polypoidal lesion with ulcerated surface and actively oozing blood in the body of stomach. 
right upper lobe and apical segment of right lower lobe with a large hypodense node in paratracheal and pretracheal regions likely metastatic and also soft tissue nodular lesions in right pelvis, stomach, mesentery, retroperitoneum and abdominal fat with large necrotic soft tissue lesion on the surface of proximal transverse colon and distal ileal loops with multiple lymph node enlargement and bilateral pleural effusion and pericardial effusion [Fig.3-5]. Fine needle aspiration from the caecal mass revealed tumor cells present in sheets with moderate amount of foamy vacuolated cytoplasm and large hyperchromatic nucleus. Patient was managed in intensive care unit with all ventilatory support. During the period of ICU stay her condition deteriorated. She had sudden cardiac arrest and could not be revived.

\section{Discussion}

Chondrosarcoma is the second most common primary bone tumor that accounts for $20 \%$ of all bone sarcomas and more common in patients over the age of 25 years [1]. The clinical course of chondrosarcoma ranges from slow growing tumor with good prognosis to aggressive behaviour with dismal prognosis which depend upon grade of tumor. Surgery is the standard of care and today the surgical procedure for the treatment of chondrosarcoma is predominantly guided by tumor grade. The most common site of metastasis is lung followed by liver. Although extremely rare, lymph nodal metastasis has also been reported in literature, lymphatic spread generally occurs when tumor extends through the periosteum into the soft tissues [2]. While high grade lesions stipulate adequate surgical margins, the management of low grade chondrosarcoma remains ill defined. The basic behavior of low grade chondrosarcoma is less aggressive clinical course, lower recurrence incidence and higher survival rate [3]. The description of low garde chondrosarcoma as an less aggressive malignancy by some authors together with its low metastatic potential have favoured a less invasive treatment at least in the long bones in form of intra-lesional curettage. Most recent reports about extremity low grade chondrosarcoma were able to confirm that intralesional surgery was not associated with higher rates of local recurrence. Additionally functional results were better and complication results lower.

In a retrospective study of 70 patients of low grade chondrosarcoma by Funovics et al.
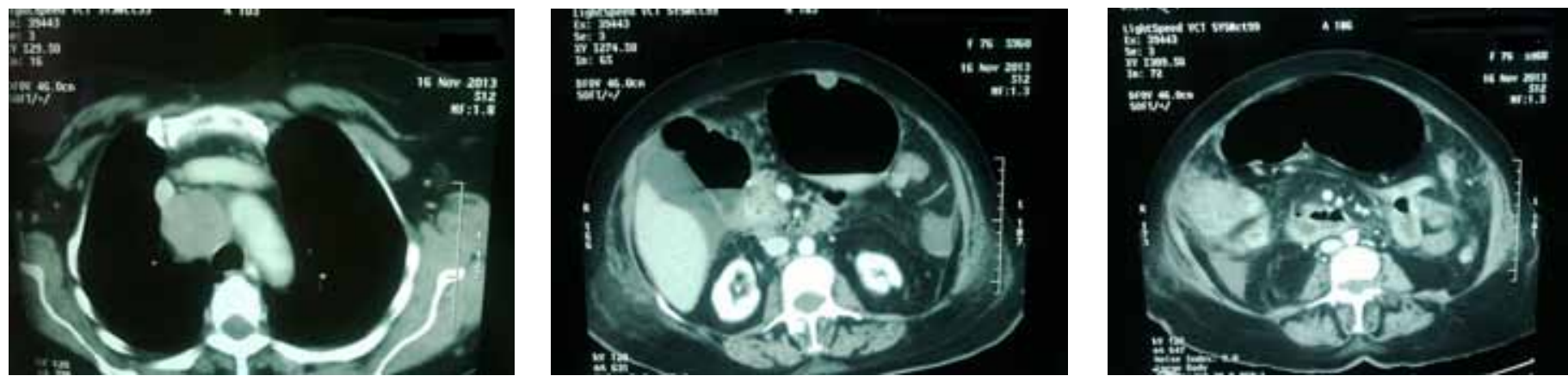

Fig.3-5: Contrast enhanced CT scan showing soft tissue density lesion in right upper lobe and lower lobe with a large hypodense node in paratracheal and pretracheal regions. Soft tissue nodular lesions in right pelvis, stomach, mesentery, retroperitoneum and abdominal fat with large necrotic soft tissue lesion on the surface of proximal transverse colon and distal ileal loops with multiple lymph node enlargement and bilateral pleural effusion and pericardial effusion. 
overall recurrence free survival of $90.6 \%$ and $86.9 \%$ at 24 months and 48 months was reported respectively, which remained constant thereafter. There was no significant difference in recurrence free survival according to resection margins at 24 months $(83.4 \%$ for intralesional resections versus $100 \%$ for marginal resections) or at 48 months $180.1 \%$ for intra-lesional resections versus $83.3 \%$ for marginal resections). They noticed $2.6 \%$ rate of distant metastasis [4]. Ozaki et al. reported that the histological grade is correlated with the metastasis rate whereas size and location has no influence on the occurrence of metastasis [5]. The stomach is an uncommon site for metastatic involvement in chondrosarcoma, the first case was reported in 1994 by Konishi et al. [6]. The most common primaries are melanoma, tumors of breast and lung.

These lesions begin in the submucosa, forming circumscribed nodules and plaques. When they get enlarge they become pedunculated and may invade mucosa producing crateriform ulcerations [7]. Yashimori et al. described the endoscopic features of metastatic tumors of stomach [8]. The route suggested for the metastatic dissemination is through lymphatic or blood borne but there are very few descriptions of the exact metastatic route followed. A number of studies have shown that prognosis of patients with isolated regional metastasis is better than patients with distant pulmonary, visceral, bone metastasis $[9,10]$. This case report is to update ourselves with the metastatic potential.

\section{Conclusion}

The aim of this report is to highlight the fact that though gastric metastasis is extremely rare in primary osseous low grade chondrosarcoma, the presence of occult blood in stool and a pedunculated mass on gastroendoscopy merits further evaluation. The presence of a nodal mass could have an impact on prognosis. The rarity of the occurrence of stomach metastasis in low grade chondrosarcoma and the scarcity of enough literature in these cases highlights the need to understand the biology and its impact on the outcome of these uncommon lesions.

\section{References}

1. Andreou D, Ruppin S, Fehlberg S, Pink D, Werner $M$, Tunn PU. Survival and prognostic factors in chondrosarcoma: results in 115 patients with long term follow up. Acta Orthop. 2011 ; 82:749 755.

2. Gulia A, Puri A, Jain S, Dhanda S, Gujral S. Chondrosarcoma of the bone with nodal metastasis:the first case report with review of literature. Indian J Med Sci. 201 1;65:360-364.

3. Lee F, Mankin RA, Fondren G, etal. Chondrosarcoma of bone: an assessment of outcome. J Bone Joint Surg Am. 1999; 81:326-338.

4. Funovics PT, Panotopoulos J, Sabeti-Aschraf M, Abdolvahab F, Funovics JM, Lang S, Kotz RI, Dominkus M. Low grade chondrosarcoma of bone: Experiences from the Vienna bone and soft tissue tumour registry. Int Orthop. 2011 ; 35:1049-1056.

5. Ozaki T, Hillmann A, Linder $\mathrm{N}$, Blasius $\mathrm{S}$, Winkelmann W. Metastasis of chondrosarcoma. J Cancer Res Clin Oncol.1996;1 22:629-632.

6. Konishi H, Isetani K, Satoh T, Fukuda S, Kodama T, Kashima K. A case of metastatic chondrosarcoma of the stomach. J Gastroenterol. 1994;29:495500.

7. Sandler RS, Sartor RB, Bozymski EM. Endoscopic appearance of cancer metastatic to the stomach. J Clin Gastroenterol. 1981 ;3:35-37.

8. Yoshimori $M$, Oka $Y$, Yashida $S$, et al. Endoscopic diagnosis of metastatic tumours of the stomach. Gastroenterol Endosc. 1977;19:821-826.

9. Raid S, Griffin AM, Liberman B, Blackstein $M E$, Catton CN, Kandel RA, et al. Lymph node metastasis in soft tissue sarcoma in an extremity. Clin Orthop Relat Res. 2004 ;426:1 29-134.

10. Al-Refaie WB, Andtbacka RH, Ensor J, Pisters PW, Ellis TL, Shrout A, Hunt KK, et al. Lymphadenectomy for isolated lymph node metastasis from extremity soft-tissue sarcomas. Cancer. 2008;112:18211826. 